\title{
Diversity and Distribution of Avifauna at the Tropical Montane Cloud Forests of Horton Plains National Park
}

\author{
P.H.S.P Chandrasiri, W.D.S.C. Dharmarathne and W.A.D Mahaulpatha* \\ Department of Zoology, University of Sri Jayewardenepura, Sri Lanka
}

Date Received: 2018-03-07

Date Accepted: 2018-04-08

\begin{abstract}
Diversity and distribution of avifauna was studied at the tropical montane cloud forests of Horton Plains National Park, situated in the highland plateau of the Nuwara Eliya district from September 2015 to May 2016. Three main habitats were identified; cloud forest habitat, cloud forest die-back habitat and grassland habitat. Nine, $300 \mathrm{~m}$ line transects were marked in each of the habitats. Avifauna was recorded on three consecutive days of each month while travelling along these transects. Seventy eight species of birds were recorded during the study period. This included 66 resident species (with 13 endemic species) and 12 migratory species. The maximum value of the Shannon Wiener Index H' of 2.56 was recorded from the cloud forest habitat. In the cloud forest die-back habitat the $\mathrm{H}^{\prime}$ was 2.49 and in the grassland habitat the $\mathrm{H}^{\prime}$ was 2.31.The Jaccard similarity index, between cloud forest and cloud forest die-back was 0.58, and these two habitats had more common species. Cloud forest is the major habitat to be protected, with other habitats, in HPNP. Hence management of the HPNP should plan more actions to improve long term monitoring plans to warrant the protection of threatened species.
\end{abstract}

Keywords: bird diversity and distribution, endemic birds, Horton Plains, tropical montane cloud forest

\section{Introduction}

Sri Lanka is a tropical island in the Indian Ocean, at the southern point of the Indian sub-continent, with a main island and several small islands. Sri Lanka is one of the eight 'Hottest Hotspots' out of the 36 Biodiversity Hotspots in the world (Myers et al., 2000). Therefore, Sri Lanka is ranked among the highest in Asia, in terms of 'biodiversity per unit area' (MOE, 2012).

Sri Lanka is rich with 453 avifaunal species recorded at present, including 240 species of breeding residents, of which 27 are endemics and six proposed endemics (Gunawardena and Weerakoon, 2012). The country is divided into six avifaunal zones, according to the distribution patterns of the resident bird species (Kotagama, 1993). These zones are northern or Indian zone, low country wet zone, mid-country wet zone, hill country wet zone, dry zone and uva zone. Hill country wet zone is one of them, which is rich and abundant with most of the endemic and threatened species (Kotagama, 1993, Harrison and Worfolk, 1999).

*Correspondence: : mahaulpatha@sjp.ac.lk

ISSN 2235-9370 Print / ISSN 2235-9362 Online ( ) University of Sri Jayewardenepura 
Furthermore, there are some threats to the avifauna in Sri Lanka. Because the forest area coverage in Sri Lanka in 2015 was $33.0 \%$, and despite the value being recorded to be, so it is fast declining (World Bank, 2016).

There is a difference in the vegetation of highland forests in Sri Lanka, above 1,500 m (Ashton et al., 1997). These forests have been classified as tropical montane cloud forests (WCMC, 1997). In Sri Lanka, Horton Plains, Hakgala Nature Reserve, Peak Wilderness Sanctuary and Knuckles are the main forests of this particular type. The Horton Plains National Park (HPNP) is a well-known place in Sri Lanka, as a saddle shaped highland plateau (Pethiyagoda, 2012) surrounded by tea and Eucalyptus plantations.

There were previous studies carried out in the HPNP, as floral and faunal inventory (MfC, 1994), National Conservation Review (Green and Gunawardena, 1997) and Management Plan (DWC, 2005). Moreover, they have studied the avifaunal diversity on that studies. However, there were no recorded study after the Biodiversity Baseline Survey at HPNP which was conducted in 2007 by Department of Wildlife Conservation. The aim of this research to fill the research gaps of avifaunal studies in the HPNP. Therefore, this study was conducted to record the avifaunal diversity and to determine the population distribution of birds in different habitats in the HPNP.

\section{Materials and Methods}

\subsection{Study area}

The study was conducted at the Horton Plains National Park $\left(6^{\circ} 47^{\prime}-6^{\circ} 50 \prime \mathrm{N}, 80^{\circ} 46^{\prime}-80^{\circ} 50^{\prime} \mathrm{E}\right)$ in Nuwara Eliya District at the eastern extremity of the central highlands (Figure 1). The HPNP is a protected area under the Department of Wildlife Conservation. The elevation of HPNP is about 2,000 m from mean sea level. The area of the national park is $31.6 \mathrm{~km}^{2}$. HPNP was divided into three main habitat types, according to different characteristics of vegetation by using the methods of Biodiversity Baseline Survey at Horton Plains National Park (DWC, 2007).

These three habitats were cloud forest, cloud forest die-back and grasslands. The cloud forest was distributed within 1,236 ha (39.7\% of total area) with an undisturbed old-growth forest which is low in height $(15-20 \mathrm{~m})$ and the canopy trees were characteristically gnarled and twisted, due to the lower temperatures and high winds. The cloud forest die-back was distributed within 956 ha $(30.7 \%$ of total area). Larger area of the canopies in the cloud forest were dead, and therefore it was known as the cloud forest die-back. There were three types of habitats which were totally considered as grasslands. These habitats were dwarf bamboo, tussock grass and carpet grass (DWC, 2007). Grassland habitat was distributed in 806 ha $(25.8 \%$ of the total area) of the national park.

\subsection{Site selection}

Sampling areas were located by using 1:50 000 analogue maps and 1:10 000 digital topographical maps, of the Department of Survey. A global positioning system device (Garmin, eTrex® 10) was used to mark transects within habitats. Nine, $300 \mathrm{~m}$ line transacts with $20 \mathrm{~m}$ swath, were marked in the cloud forest, cloud forest die-back and grassland habitats. 


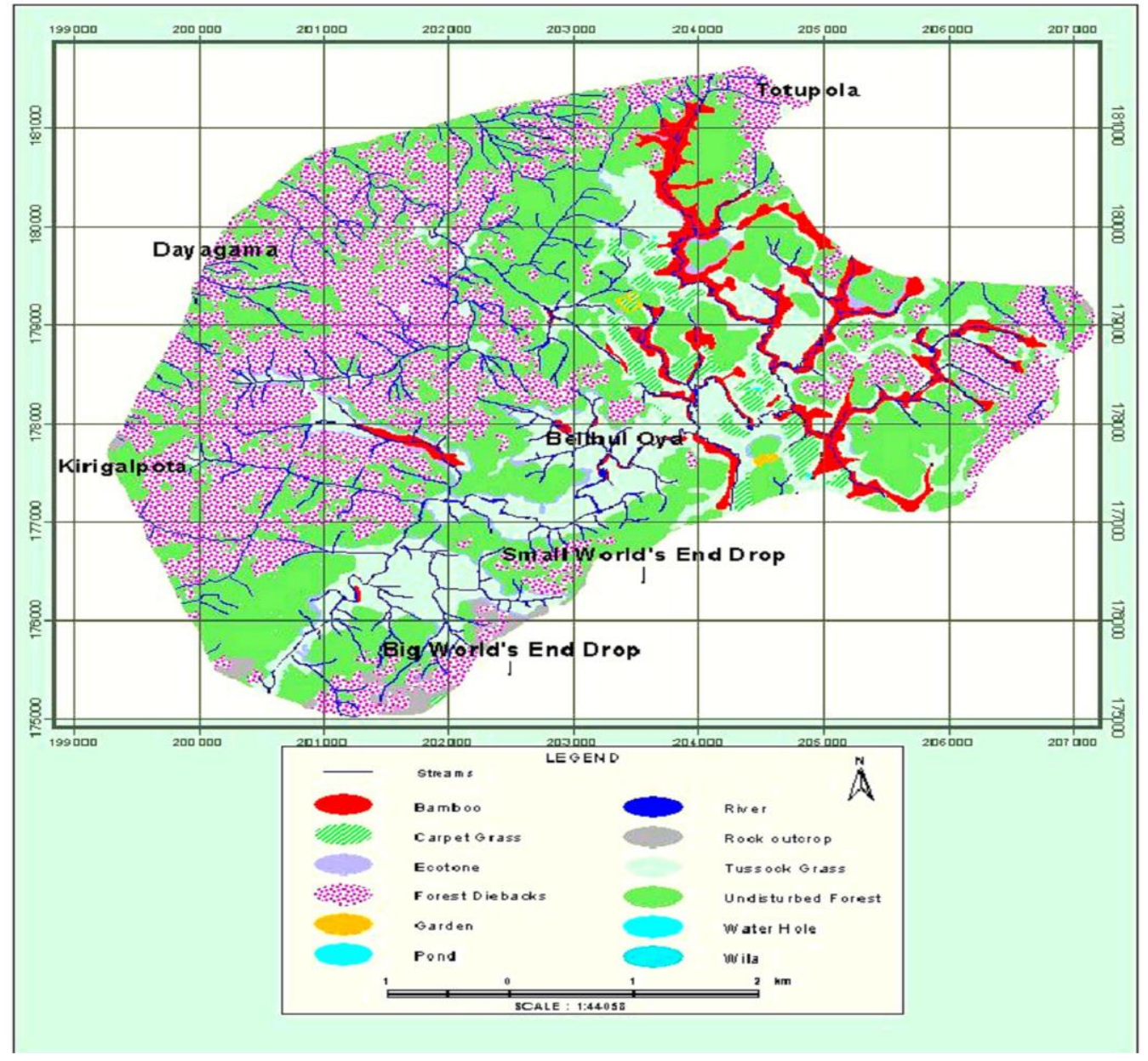

Figure 1: Vegetation map of Horton Plains National Park (Source: DWC, 2007).

\subsection{Data collection}

All the species of birds seen or heard within transect, were identified by using a 10x50 binocular (Nikon, Monarch) and recorded by using field guides of Harrison and Worfolk (1999). Identified bird species were classified according to the National Red List of 2012 (MOE, 2012) and the Revised Avifaunal List of Sri Lanka (Kaluthota and Kotagama, 2009) Population of the birds was recorded on three consecutive days of each month, from September 2015 to May 2016 by traveling along transacts, from 05.30 a.m. to 09.30 a.m. Finally, opportunistic data and incidental observations were used to supplement information (Sutherland et al., 2004).

\subsection{Data analysis}

Relative abundance [(number of individuals per species/total number of individuals)x 100\%], Shannon Weiner Diversity Index H' was calculated using the equation 1.

$$
H^{\prime}=-\sum\left(P_{i} \ln P_{i}\right)
$$

Where;

$\mathrm{P}_{\mathrm{i}}=$ the proportion of the total sample belonging to the $\mathrm{i}^{\mathrm{th}}$ species to measure the avifaunal diversity 
Significant differences between the monthly diversity indices were calculated using the $\mathrm{t}$ test. [ $\mathrm{t}=$ $\left(\mathrm{H}^{\prime}-\mathrm{H}\right) /\left(\operatorname{varH}_{1}+\right.$ var $\left.\left.\mathrm{H}_{2}\right) 1 / 2\right]$ (Magurran, 1988). Jaccard Similarity Index $\{\mathrm{SJ}\}$ was calculated between the habitat by using the equation 2 .

$$
\mathrm{SJ}=\mathrm{Sa} /(\mathrm{Sa}+\mathrm{Sb}+\mathrm{Sc})
$$

Where;

$\mathrm{Sa}=$ number of species unique to the first habitat

$\mathrm{Sb}=$ number of the species unique to the second habitat

$\mathrm{Sc}=$ number of species common in both habitats

Microsoft Excel ${ }^{\mathrm{TM}}$ was used to store data and further more calculations and illustrations of the figures. Minitab $17^{\mathrm{TM}}$ was used for statistical analysis of variance and $\mathrm{t}$ - test.

\section{Results}

Although there were 31 species recorded in the first month, this value was increased to seventy eight by the ninth month. Furthermore, the cumulative number of the species was recorded as a plateau in the last five months (Figure 2). Therefore, it was determined that the sampling effort was adequate for final calculations and analysis. Seventy eight species within 33 families of birds were recorded during the study period (Table 1). This included 66 resident species (with 13 endemic species) and 12 migratory species. A total of 4,537 individuals belonging to 60 species were recorded in the cloud forest habitat. 1,870 individuals belonging to 45 species were recorded in the Cloud forest die-back habitat. Moreover 2,897 individuals that belong to 41 species were recorded in the grassland habitat.

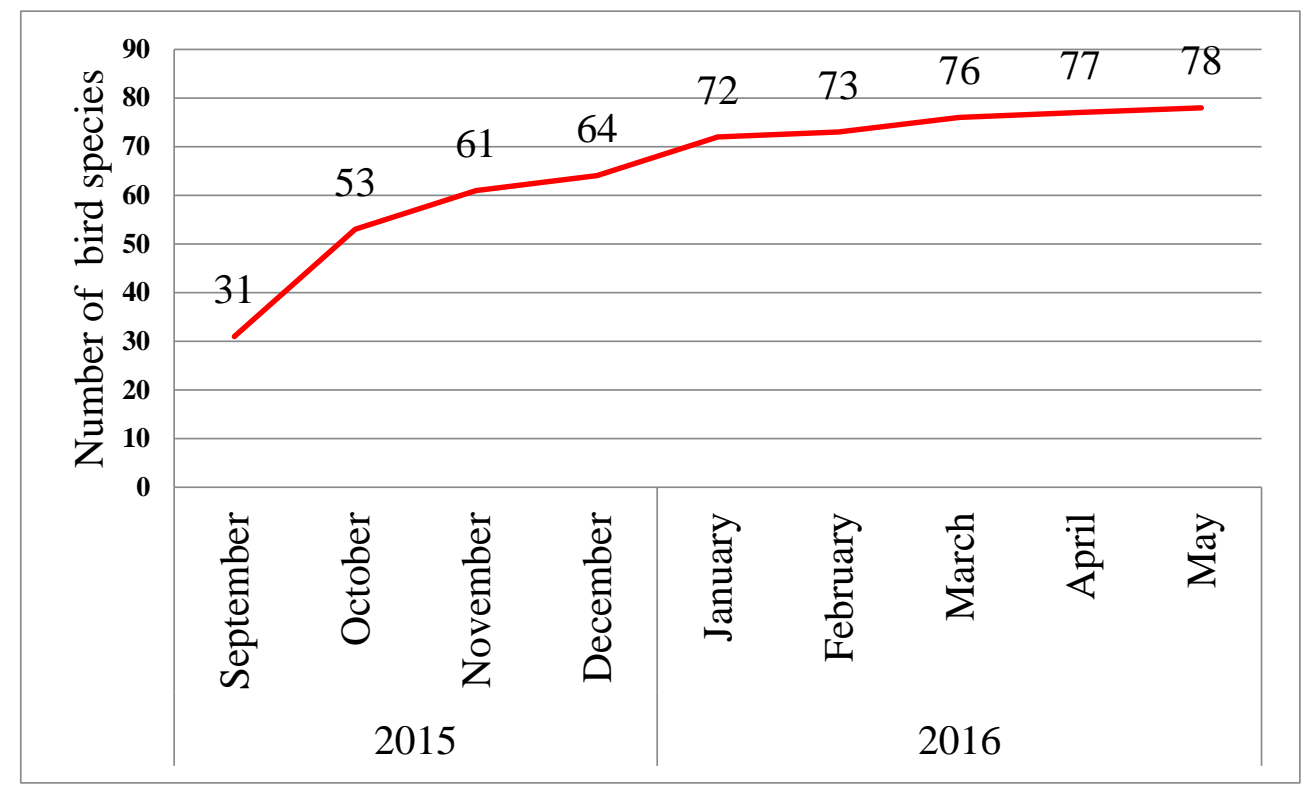

Figure 2: Cumulative number of bird species. 
Table 1: Resident bird species and total number of species recorded during the study period.

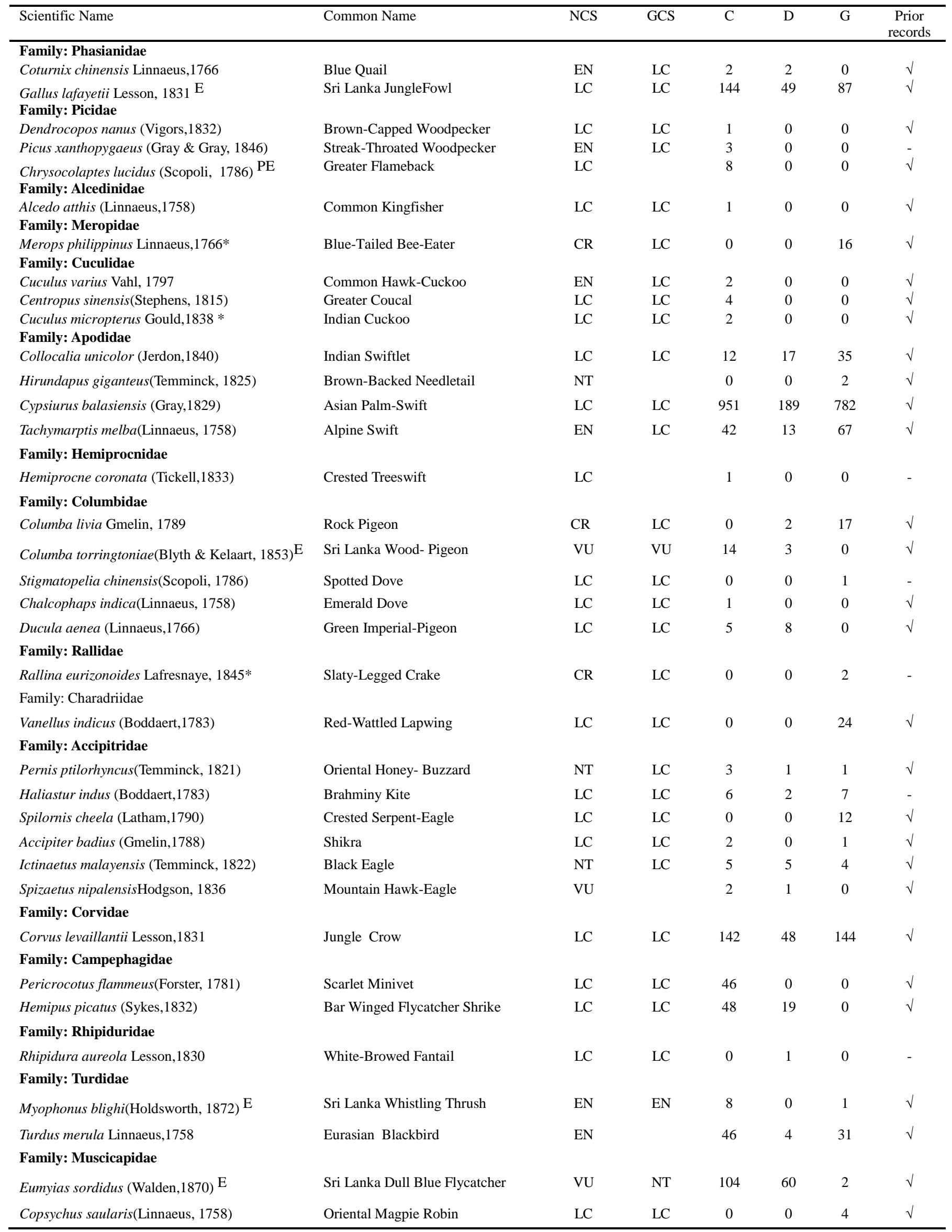


Chandrasiri et al. /Journal of Tropical Forestry and Environment Vol. 8, No. 01 (2018) 36-49

\begin{tabular}{|c|c|c|c|c|c|c|c|}
\hline Saxicola caprata (Linnaeus,1766) & Pied Bush Chat & EN & $\mathrm{LC}$ & 3 & 25 & 188 & $\sqrt{ }$ \\
\hline Culicicapa ceylonensis Swainson, 1820 & Grey- Headed Canary- Flycatcher & $\mathrm{LC}$ & $\mathrm{LC}$ & 37 & 0 & 0 & $\sqrt{ }$ \\
\hline \multicolumn{8}{|l|}{ Family: Sturnidae } \\
\hline Gracula ptilogenys Blyth, $1846^{\mathrm{E}}$ & Sri Lanka Myna & VU & NT & 2 & 0 & 0 & $\sqrt{ }$ \\
\hline \multicolumn{8}{|l|}{ Family: Sittidae } \\
\hline Pavus major Linnaeus, 1758 & Great Tit & $\mathrm{LC}$ & & 68 & 51 & 0 & $\sqrt{ }$ \\
\hline \multicolumn{8}{|l|}{ Family: Hirundinidae } \\
\hline Hirundo domicola Jerdon, 1844 & Hill Swallow & VU & $\mathrm{LC}$ & 564 & 173 & 819 & $\sqrt{ }$ \\
\hline \multicolumn{8}{|l|}{ Family: Pycnonotidae } \\
\hline Cisticola juncidis (Rafinesque,1810) & Zitting Cisticola & $\mathrm{LC}$ & $\mathrm{LC}$ & 13 & 2 & 84 & $\sqrt{ }$ \\
\hline Prinia socialis Sykes, 1832 & Ashy Prinia & $\mathrm{LC}$ & $\mathrm{LC}$ & 3 & 11 & 0 & - \\
\hline \multicolumn{8}{|l|}{ Family: Zosteropidae } \\
\hline Zosterops ceylonensis Holdsworth, $1872 \mathrm{E}$ & Sri Lanka White Eye & NT & $\mathrm{LC}$ & 797 & 447 & 16 & $\sqrt{ }$ \\
\hline \multicolumn{8}{|l|}{ Family: Sylviidae } \\
\hline Bradypterus palliseri (Blyth,1851) $\mathrm{E}$ & Sri Lanka Bush Warbler & EN & NT & 25 & 23 & 0 & $\sqrt{ }$ \\
\hline Orthotomus sutorius(Pennant, 1769) & Common Tailorbird & $\mathrm{LC}$ & LC & 41 & 7 & 0 & $\sqrt{ }$ \\
\hline \multicolumn{8}{|l|}{ Family: Timaliidae } \\
\hline Garrulax cinereifrons Blyth, $1851^{\mathrm{E}}$ & $\begin{array}{l}\text { Sri Lanka Ashy-Headed } \\
\text { Laughingthrush }\end{array}$ & EN & VU & 5 & 4 & 0 & $\sqrt{ }$ \\
\hline Chrysomma sinense (Gmelin,1789) & Yellow Eyed Babbler & $\mathrm{LC}$ & $\mathrm{LC}$ & 1 & 0 & 0 & - \\
\hline Turdoides rufescens (Blyth,1847) $\mathrm{E}$ & Sri Lanka Orange Billed Babbler & VU & NT & 17 & 0 & 0 & $\sqrt{ }$ \\
\hline Turdoides affinis (Jerdon, 1845) & Yellow Billed Babbler; & $\mathrm{LC}$ & $\mathrm{LC}$ & 2 & 9 & 2 & $\sqrt{ }$ \\
\hline \multicolumn{8}{|l|}{ Family: Dicaeidae } \\
\hline Dicaeum erythrorhynchos(Latham, 1790) & Pale Billed Flowerpecker & $\mathrm{LC}$ & $\mathrm{LC}$ & 414 & 108 & 23 & $\sqrt{ }$ \\
\hline \multicolumn{8}{|l|}{ Family: Nectariniidae } \\
\hline Nectarinia asiatica (Latham,1790) & Purple Sunbird & $\mathrm{LC}$ & $\mathrm{LC}$ & 4 & 4 & 0 & $\sqrt{ }$ \\
\hline Nectarinia lotenia (Linnaeus,1766) & Long Billed Sunbird & $\mathrm{LC}$ & $\mathrm{LC}$ & 24 & 8 & 11 & $\sqrt{ }$ \\
\hline Nectarinia zeylonica (Linnaeus, 1766) & Purple Rumped Sunbird & $\mathrm{LC}$ & $\mathrm{LC}$ & 4 & 3 & 0 & $\sqrt{ }$ \\
\hline \multicolumn{8}{|l|}{ Family: Motacillidae } \\
\hline Anthus rufulus Vieillot, 1818 & Paddyfield Pipit & $\mathrm{LC}$ & $\mathrm{LC}$ & 0 & 7 & 105 & $\sqrt{ }$ \\
\hline \multicolumn{8}{|l|}{ Family: Estrildidae } \\
\hline Lonchura striata (Linnaus,1766) & White Rumped Munia & $\mathrm{LC}$ & $\mathrm{LC}$ & 0 & 0 & 22 & $\sqrt{ }$ \\
\hline Lonchura punctulata(Linnaeus, 1758) & Scaly Breasted Munia & $\mathrm{LC}$ & $\mathrm{LC}$ & 0 & 2 & 55 & $\sqrt{ }$ \\
\hline Lonchura malacca (Linnaeus,1766) & Tricoloured Munia & $\mathrm{LC}$ & $\mathrm{LC}$ & 5 & 13 & 242 & $\sqrt{ }$ \\
\hline
\end{tabular}


Maximum number of individuals was the hills wallow followed by the Asian palm swift. The highest number of species was recorded from the Family Timaliidae (babblers). The most significant about these findings was that all the species of that family whereas present at Horton Plains National Park.

Table 2: Migratory birds and vagrants species recorded during the study period. (* Only the breeding population has been considered in this assessment)

\begin{tabular}{|c|c|c|c|c|c|c|c|}
\hline Scientific Name & Common Name & NCS & GCS & $\mathrm{C}$ & $\mathrm{D}$ & $\mathrm{G}$ & $\begin{array}{c}\text { Prior } \\
\text { Records } \\
\end{array}$ \\
\hline $\begin{array}{l}\text { Family: Muscicapidae } \\
\text { Ficedula subrubra(Hartert \& } \\
\text { Steinbacher, 1934) }\end{array}$ & Kashmir Flycatcher & & VU & 4 & 0 & 0 & $\sqrt{ }$ \\
\hline Muscicapa dauurica Pallas, 1811 & $\begin{array}{l}\text { Asian Brown } \\
\text { Flycatcher }\end{array}$ & & LC & 0 & 4 & 0 & $\sqrt{ }$ \\
\hline Muscicapa muttui (Layard, 1854) & $\begin{array}{l}\text { Brown-Breasted } \\
\text { Flycatcher }\end{array}$ & & $\mathrm{LC}$ & 1 & 0 & 0 & - \\
\hline $\begin{array}{l}\text { Luscinia brunnea (Hodgson, 1837) } \\
\text { Family: Laniidae }\end{array}$ & Indian Blue Robin & & $\mathrm{LC}$ & 1 & 0 & 0 & $\sqrt{ }$ \\
\hline $\begin{array}{l}\text { Lanius cristatus Linnaeus, } 1758 \\
\text { Family: Motacillidae }\end{array}$ & Brown Shrike & & $\mathrm{LC}$ & 3 & 3 & 8 & $\sqrt{ }$ \\
\hline Dendronanthus indicu s (Gmelin, 1789) & Forest Wagtail & & LC & 9 & 2 & 7 & $\sqrt{ }$ \\
\hline Motacilla flava Linnaeus, 1758 & Yellow Wagtail & & $\mathrm{LC}$ & 7 & 0 & 1 & - \\
\hline $\begin{array}{l}\text { Motacilla cinerea Tunstall, } 1771 \\
\text { Family: Hirundinidae }\end{array}$ & Grey Wagtail & & LC & 12 & 8 & 25 & $\sqrt{ }$ \\
\hline Hirundo rustica Linnaeus, 1758 & Barn Swallow & & LC & 3 & 8 & 11 & $\sqrt{ }$ \\
\hline $\begin{array}{l}\text { Riparia riparia (Linnaeus, 1758) } \\
\text { Family: Laridae }\end{array}$ & Sand Martin & & $\mathrm{LC}$ & 0 & 0 & 7 & - \\
\hline $\begin{array}{l}\text { Chlidonias hybrida (Pallas, 1811) } \\
\text { Family Scolopacidae }\end{array}$ & Whiskered Tern & & $\mathrm{LC}$ & 0 & 0 & 1 & - \\
\hline Gallinago stenura (Bonaparte, 1830) & Pintail Snipe & & $\mathrm{LC}$ & 0 & 0 & 2 & $\sqrt{ }$ \\
\hline
\end{tabular}

Abbreviations: $\mathrm{C}=\mathrm{Cloud}$ Forest, $\mathrm{D}=$ Cloud Forest Die-back, E=Endemic species, G=Grassland, GCS=Global Conservation Status, NCS=National Conservation Status, $\mathrm{PE}=$ Possibly Endemic

Critically Endangered (CR), Endangered (EN), Vulnerable (VU), Near Threatened (NT), Least Concern (LC)

Out of the 12 species from the recorded migratory birds at HPNP, the highest number of species were recorded from Family Muscicapidae (Table 2). Moreover three species of wagtails were recorded. In addition aquatic birds; whiskered tern and pintail snipe were recorded. Of the 78 species that were included for this research there were ten species, which only one individual was recorded (Table 1 and 2). Lowland common species such as the Common Kingfisher and the Spotted Dove were recorded in the least numbers.

A total of 19 species of birds that were believed to be nationally endangered, were recorded within the study period. Of them Blue-Tailed Bee-Eater, Rock Pigeon (taking into consideration only the wild population for this purpose) and Slaty-Legged Crake were in the CR (Critically Endangered) category. Besides Blue Quail, Streak-Throated Woodpecker, Common Hawk-Cuckoo, Alpine Swift, Sri Lanka Whistling Thrush, Eurasian Blackbird, Pied Bush Chat, Sri Lanka Bush Warbler and Sri Lanka AshyHeaded Laughing thrush were in the EN (Endangered) category. Additionally, Sri Lanka Wood Pigeon, Mountain Hawk-Eagle, Sri Lanka Dull Blue Flycatcher, Sri Lanka Myna, Hill Swallow, Sri Lanka Yellow-Eared Bulbul and Sri Lanka Orange Billed Babbler were in the VU (Vulnerable) category. 
There were globally threatened species were recorded: Sri Lanka Whistling Thrush (EN), Sri Lanka Wood Pigeon (VU), Sri Lanka Ashy-headed Laughing thrush (VU) and the Kashmir Flycatcher (VU). There were eight new breeding resident species (Streak-throated Woodpecker, Crested Treeswift, Spotted Dove, Slaty-Legged Crake, Brahminy Kite, White-Browed Fantail, Ashy Prinia, Tawny-Bellied Babbler and Yellow-Eyed Babbler) and four migratory species (Brown-breasted Flycatcher, Yellow Wagtail, Sand Martin and Whiskered Tern) recorded for the first time in HPNP. Of the species of birds which were recorded from the fixed line transects, only 35 species were from the cloud forest while 26 species were from the cloud forest die-back and 23 species from the grassland. Therefore these species were only subjected to statistical analysis, while the other recorded species were treated as opportunistic observations.

Table 3: Relative Abundance of cloud forest.

\begin{tabular}{|c|c|c|}
\hline Rank & Species & Relative Abundance $\%$ \\
\hline 1 & Asian Palm Swift & 20.96 \\
\hline 2 & Sri Lanka White-Eye & 17.56 \\
\hline 3 & Sri Lanka Yellow-Eared Bulbul & 13.26 \\
\hline 4 & Hill Swallow & 12.43 \\
\hline 5 & Pale Billed Flowerpecker & 9.12 \\
\hline 6 & Sri Lanka Junglefowl & 3.17 \\
\hline 7 & Jungle Crow & 3.12 \\
\hline 8 & Velvet-Fronted Nuthatch & 2.64 \\
\hline 9 & Sri Lanka Dull-Blue Flycatcher & 2.29 \\
\hline 10 & Dark-Fronted Babbler & 1.89 \\
\hline 11 & Great Tit & 1.49 \\
\hline 12 & Sri Lanka Scimitar Babbler & 1.10 \\
\hline 13 & Bar-Winged Flycatcher-Shrike & 1.05 \\
\hline 14 & Scarlet Minivet & 1.02 \\
\hline 15 & Eurasian Blackbird & 1.01 \\
\hline 16 & Alpine Swift & 0.92 \\
\hline 17 & Common Tailorbird & 0.90 \\
\hline 18 & Grey-Headed Canary Flycatcher & 0.81 \\
\hline 19 & Sri Lanka Bush Warbler & 0.55 \\
\hline 20 & Long-Billed Sunbird & 0.52 \\
\hline 21 & Sri Lanka Orange-Billed Babbler & 0.37 \\
\hline 22 & Sri Lanka Woodpigeon & 0.30 \\
\hline 23 & Zitting Cisticola & 0.28 \\
\hline 24 & Indian Swiftlet & 0.26 \\
\hline 25 & Grey Wagtail & 0.26 \\
\hline 26 & Forest Wagtail & 0.19 \\
\hline 27 & Sri Lanka Whistling-Thrush & 0.17 \\
\hline 28 & Greater Flameback & 0.17 \\
\hline 29 & Yellow Wagtail & 0.15 \\
\hline 30 & Brahminy Kite & 0.13 \\
\hline 31 & Black Eagle & 0.11 \\
\hline 32 & $\begin{array}{l}\text { Sri Lanka Ashy-Headed Laughing } \\
\text { Thrush }\end{array}$ & 0.11 \\
\hline 33 & Common Myna & 0.11 \\
\hline 34 & Black-Headed Munia & 0.11 \\
\hline 35 & Green Imperial Pigeon & 0.11 \\
\hline
\end{tabular}


Table 4: Relative abundance of the cloud forest die-back.

\begin{tabular}{llc}
\hline Rank & Common Name & $\begin{array}{c}\text { Relative } \\
\text { Abundance } \%\end{array}$ \\
\hline 1 & Sri Lanka White-Eye & 23.90 \\
2 & Sri Lanka Yellow-Eared Bulbul & 23.79 \\
3 & Asian Palm Swift & 10.10 \\
4 & Hill Swallow & 9.25 \\
5 & Pale-Billed Flower-Pecker & 5.77 \\
6 & Sri Lanka Dull-Blue Flycatcher & 3.20 \\
7 & Dark-Fronted Babbler & 2.72 \\
8 & Great Tit & 2.72 \\
9 & Sri Lanka Junglefowl & 2.62 \\
10 & Jungle Crow & 2.56 \\
11 & Pied Bushchat & 1.33 \\
12 & Sri Lanka Bush Warbler & 1.22 \\
13 & Bar-Winged Flycatcher-Shrike & 1.01 \\
14 & Sri Lanka Scimitar Babbler & 1.01 \\
15 & Indian Swiftlet & 0.90 \\
16 & Black-Headed Munia & 0.69 \\
17 & Alpine Swift & 0.69 \\
18 & Velvet-Fronted Nuthatch & 0.53 \\
19 & Yellow-Billed Babbler & 0.48 \\
20 & Red-Vented Bulbul & 0.48 \\
21 & Barn Swallow & 0.42 \\
22 & Long-Billed Sunbird & 0.42 \\
23 & Grey Wagtail & 0.42 \\
24 & Green Imperial Pigeon & 0.42 \\
25 & Paddyfield Pipit & 0.37 \\
26 & Common Tailorbird & 0.37 \\
\hline & &
\end{tabular}

According to the relative abundance values in the cloud forest habitat (Table 3) the Asian Palm Swift was the most common bird (relative abundance=20.96\% ) followed by the Sri Lanka White-Eye, the Sri Lanka Yellow-Eared Bulbul, the Hill Swallow and the Pale-Billed Flowerpecker. In the cloud forest die-back habitat (Table 4) the most common species was the Sri Lanka White-Eye (relative abundance $=23.90 \%$ ) followed by the Asian Palm Swift, the Black-Headed Munia, the Pied Bush Chat and the Jungle Crow.

In the cloud forest die-back habitat (Table 5), the most common species was the Hill Swallow (relative abundance=28.2706) followed by the Asian Palm Swift, the Black-Headed Munia, the Pied Bush Chat and the Jungle Crow. 
Table 5: Relative abundance of grassland.

\begin{tabular}{clc}
\hline Rank & Common Name & $\begin{array}{c}\text { Relative } \\
\text { Abundance \% }\end{array}$ \\
\hline 1 & Hill Swallow & 28.27 \\
2 & Asian Palm Swift & 26.99 \\
3 & Black-Headed Munia & 8.35 \\
4 & Pied Bushchat & 6.48 \\
5 & Jungle Crow & 4.97 \\
6 & Paddyfield Pipit & 3.62 \\
7 & Sri Lanka Junglefowl & 3.01 \\
8 & Zitting Cisticola & 2.89 \\
9 & Alpine Swift & 2.31 \\
10 & Scaly-Breasted Munia & 1.89 \\
11 & Indian Swiftlet & 1.20 \\
12 & Common Myna & 1.17 \\
13 & Eurasian Blackbird & 1.07 \\
14 & Grey Wagtail & 0.86 \\
15 & Red-Wattled Lapwing & 0.82 \\
16 & Pale-Billed Flowerpecker & 0.79 \\
17 & White-Rumped Munia & 0.75 \\
18 & Rock Pigeon & 0.58 \\
19 & Sri Lanka White-Eye & 0.55 \\
20 & Blue Tailed Bee-Eater & 0.55 \\
21 & Crested Serpent Eagle & 0.41 \\
22 & Barn Swallow & 0.37 \\
23 & Brown Shrike & 0.27 \\
\hline & &
\end{tabular}

From the total species taken into account in this study, 50 species of birds (Table 06) were observed opportunistically, while some rare species were also recorded in least numbers and a special feature being, the record of the Oriental Honey-Buzzard in all three habitats.

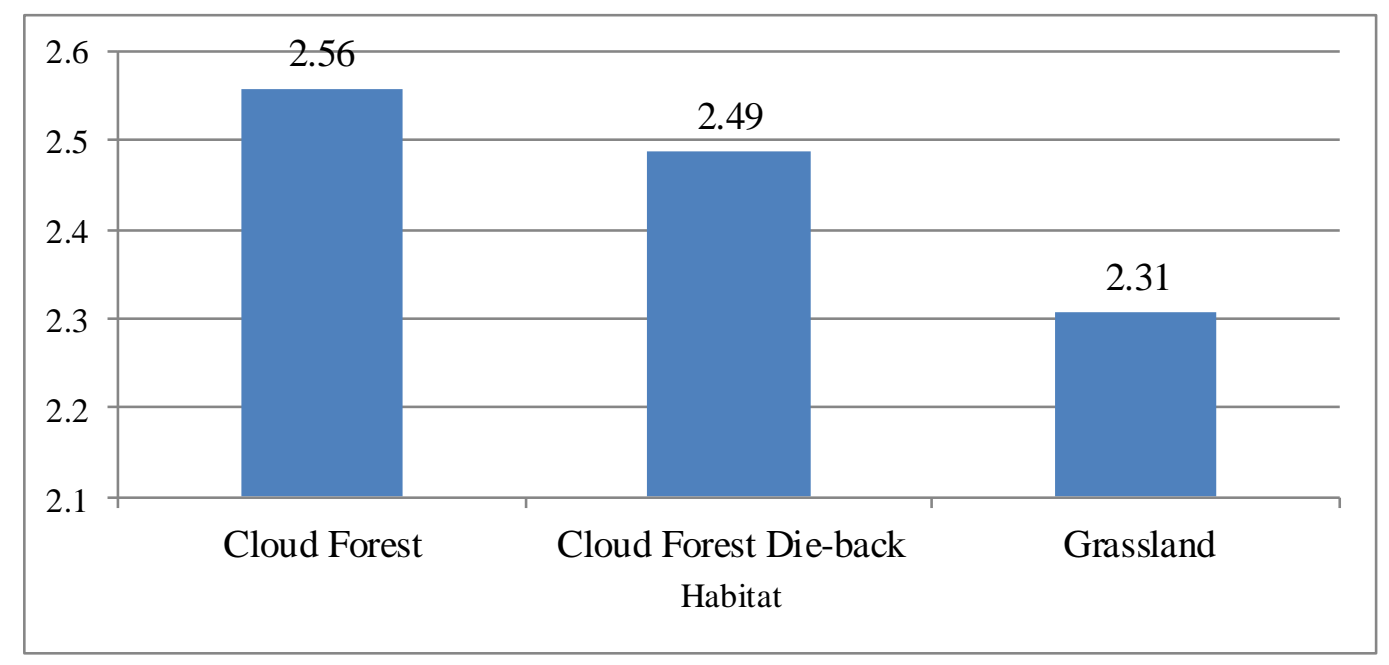

Figure 3: Shannon Wiener Index of the main three Habitats of Horton Plains. 
Table 6: Opportunistic data recorded at three habitats.

\begin{tabular}{|c|c|c|c|c|c|}
\hline Number & Common Name & $\begin{array}{l}\text { Cloud } \\
\text { forest }\end{array}$ & $\begin{array}{c}\text { Cloud forest } \\
\text { die-back }\end{array}$ & Grassland & Total \\
\hline 1. & Kashmir Flycatcher & 4 & - & - & 4 \\
\hline 2. & Greater Coucal & 4 & - & - & 4 \\
\hline 3. & Purple Sunbird & 4 & 4 & - & 8 \\
\hline 4. & Purple Rumped Sunbird & 4 & 3 & - & 7 \\
\hline 5. & Pied Bushchat & 3 & - & - & 3 \\
\hline 6. & Brown-Capped Babbler & 3 & - & - & 3 \\
\hline 7. & Oriental Honey-buzzard & 3 & 1 & 1 & 5 \\
\hline 8. & Barn Swallow & 3 & - & - & 3 \\
\hline 9. & Ashy Prinia & 3 & 1 & - & 4 \\
\hline 10. & Streaked-Throated Woodpecker & 3 & - & - & 3 \\
\hline 11. & Brown Shrike & 3 & 3 & - & 6 \\
\hline 12. & Yellow-Billed Babbler & 2 & - & 2 & 4 \\
\hline 13. & Sri Lanka Hill Myna & 2 & - & - & 2 \\
\hline 14. & Shikra & 2 & - & 1 & 3 \\
\hline 15. & Indian Cucoo & 2 & - & - & 2 \\
\hline 16. & Mountain Hawk Eagle & 2 & 1 & - & 3 \\
\hline 17. & Common Hawk Cucoo & 2 & - & - & 2 \\
\hline 18. & Blue-Breasted quail & 2 & - & 2 & 4 \\
\hline 19. & Yellow-Eyed Babbler & 1 & - & - & 1 \\
\hline 20. & Indian Blue Robin & 1 & - & - & 1 \\
\hline 21. & Emerald Dove & 1 & - & - & 1 \\
\hline 22. & Brown-breasted Flycatcher & 1 & - & - & 1 \\
\hline 23. & Crested Treeswift & 1 & - & - & 1 \\
\hline 24. & Tawny Bellied Babbler & 1 & 1 & 0 & 4 \\
\hline 25. & Common Kingfisher & 1 & - & - & 1 \\
\hline 26. & Brown-Capped Pygmy Woodpecker & 1 & - & - & 1 \\
\hline 27. & Black Eagle & - & 5 & 4 & 9 \\
\hline 28. & Eurasian Blackbird & - & 4 & - & 4 \\
\hline 29. & $\begin{array}{l}\text { Sri Lanka Ashyheaded Laughing } \\
\text { Thrush }\end{array}$ & - & 4 & - & 4 \\
\hline 30. & Asian Brown Flycatcher & - & 4 & - & 4 \\
\hline 31. & Sri Lanka Woodpigeon & - & 3 & - & 3 \\
\hline 32. & Scaly-Breasted Munia & - & 2 & - & 2 \\
\hline 33. & Forest Wagtail & - & 2 & 7 & 9 \\
\hline 34. & Rock Pigeon & - & 2 & - & 2 \\
\hline 35. & Common Myna & - & 2 & - & 2 \\
\hline 36. & Brahminy Kite & - & 2 & 7 & 9 \\
\hline 37. & Zitting Cisticola & - & 2 & - & 2 \\
\hline 38. & White-browed Fantail Flycatcher & - & 1 & - & 1 \\
\hline 39. & Sand Martin & - & - & 7 & 7 \\
\hline 40. & Oriental Magpie Robbin & - & - & 4 & 4 \\
\hline 41. & Red-vented Bulbul & - & - & 4 & 4 \\
\hline 42. & Sri Lanka Dull-blue Flycatcher & - & - & 2 & 2 \\
\hline 43. & Slaty-legged Crake & - & - & 2 & 2 \\
\hline 44. & Brown-Breasted Needle-tail & - & - & 2 & 2 \\
\hline 45. & Pintail Snipe & - & - & 2 & 2 \\
\hline 46. & Yellow Wagtail & - & - & 1 & 1 \\
\hline 47. & Sri Lanka Whistling thrush & - & - & 1 & 1 \\
\hline 48. & Spotted Dove & - & - & 1 & 1 \\
\hline 49. & Long-billed Sunbird & - & - & 1 & 1 \\
\hline 50. & Whiskered Tern & - & - & 1 & 1 \\
\hline
\end{tabular}


Maximum Shannon Wiener Index $\left(\mathrm{H}^{\prime}\right)$ was recorded from cloud forest habitat $\left(\mathrm{H}^{\prime}=2.56\right)$ while a minimum $\mathrm{H}^{\prime}$ was recorded from grassland habitat $\left(\mathrm{H}^{\prime}=2.31\right)$. Moreover after applying the $\mathrm{t}$-test, in the cloud forest die-back habitat, the $\mathrm{H}^{\prime}$ is 2.4 (Figure 3). Bird diversity differed significantly between the cloud forest die-back habitat and the grassland habitat $(\mathrm{t}=2.2587, \mathrm{df}=3826)$ as well as between the cloud forest and grassland habitats $(\mathrm{t}=3.9484, \mathrm{df}=6484)$. Bird diversity of the cloud forest did not differ significantly from the cloud forest die-back bird diversity $(\mathrm{t}=0.9325, \mathrm{df}=3529)$.

Table 7: Jaccard Similarity Index.

\begin{tabular}{lccccc}
\hline Habitat & $\mathrm{Sa}$ & $\mathrm{Sb}$ & $\mathrm{Sc}$ & $\mathrm{Sum}$ & Jaccard Index \\
\hline Cloud Forest; Cloud forest die-back & 22 & 6 & 38 & 66 & 0.58 \\
Cloud forest die-back; Grassland & 17 & 14 & 27 & 58 & 0.47 \\
Cloud Forest ;Grassland & 24 & 15 & 26 & 65 & 0.40 \\
\hline
\end{tabular}

Jaccard Similarity Index (Table 7) between the cloud forest and cloud forest die-back was at 0.58 and these habitats had more similar species. Moreover between the cloud forest die-back and the grasslands, the value was at 0.47 and minimum value, 0.40 was recorded between the cloud forest and the grasslands which had less similar species.

\section{Discussion}

Though the Horton Plains National Park is relatively smaller than other National parks (DWC, 2007), this area is rich in avifaunal diversity with a large number of bird species (Total number of species=78). There are endemic bird species with high population, present within the HPNP. Within the study there were some bird species that were not recorded in any of the previous surveys. They consisted of resident breeders such as, the Streak-Throated Woodpecker, the Crested Tree Swift, the Spotted Dove, the SlatyLegged Crake, the Brahminy Kite, the White-Browed Fantail, the Ashy Prinia, the Tawny-Bellied Babbler and the Yellow-Eyed Babbler. Moreover there were migratory species such as the BrownBreasted Flycatcher, Yellow Wagtail, Sand Martin and the Whiskered Tern.

In this study, percentage of the Sri Lankan breeding residents, 30.19\% were recorded. Furthermore out of the endemic birds (27 considered endemic birds according to the National Red List: MOE, 2012) there were $48.15 \%$ were from Horton Plains National Park. Although the Rock Pigeon (Threatened category:CR) had recoded within the study, however, they were recorded near habitats which were disturbed by humans. Therefore, this pigeons should be feral pigeons (Columba livia intermedia) which were in LC category.

In previous diversity studies done by floral and faunal inventory (MFC, 1994) they have mentioned 87 bird species with 14 endemic species. In addition National Conservation Review (Green and Gunawardena, 1997) has recorded 26 species with 5 endemic birds. Furthermore Management Plan (DWC, 2005) has mentioned 87 species with 14 endemics. Final study about the bird diversity done by the Department of Wildlife Conservation has recorded 64 species with 13 endemic birds (DWC, 2007).

Out of the newly recorded species, though the Slaty-Legged Crake was previously not recorded from Horton Plains National Park. However they were appeared in Victoria Park of Nuwara Eliya (Pethiyagoda, 2012). On the contrary, common bird species within lowlands such as Spotted Dove and 
Brahminy Kite were also observed by present study. Sand Martin was an irregular visitor (Vagrant) and a migratory species, recorded for the first time.

There is a significant difference of diversity and distribution of birds, among three habitats. There were more common bird species recorded from the cloud forest and cloud forest die-back habitats. This may be because of there was only little distinction between these two habitats in terms of floristic characteristics (DWC, 2007).

Habitat preference is highest in the cloud forest, according to the species richness (60) and species diversity (Shannon Wiener index=2.56). Cloud forest is the major habitat to be protected, with other habitats, in HPNP.

Furthermore, in this study some previously recorded (MfC, 1994) bird species were not observed. Night birds such as, Spot-Bellied Eagle Owl (Bubo nipalensis, Hodgson, 1836), was not observed because this study was carried out only in the morning period. Moreover, some species such as the Black-Winged Kite (Elanus caeruleus, Desfontaines, 1789), Montagu's Harrier (Circus pygargus, Linnaeus, 1758), Crested Goshawk (Accipiter trivirgatus, Temminck, 1824), Booted Eagle (Hieraaetus pennatus, Gmelin, 1788) and Common Kestrel (Falco tinnunculus, Linnaeus, 1758) were not recorded because of the time limitation. In addition, endemic birds such as Sri Lanka Spurfowl (Galloperdix bicalcarata, Forster, 1781) and Sri Lanka Grey Hornbill (Ocyceros gingalensis, Shaw, 1811) was not recorded in this study. However, the study is ongoing and the authors will publish their new findings in near future.

\section{Conclusion}

Present study reveals about the diversity and population distribution of the bird species at the Horton Plains National Park. Hence management of the HPNP should plan more actions to improve further long term monitoring plans of avifauna to warrant the protection by minimizing threats. The HPNP is a protected area, however there are other plantations around this area. Therefore, it is important to encourage the surrounding plantations to improve resources by practicing environmental friendly performances, such as to develop mixed plantings of Eucalyptus with other native fast-growing species (Williams, 2015).

\section{Acknowledgement}

The authors wish to thank research grant of University of Sri Jayewardenepura (ASP/01/RE/SCI/2015/34), Department of Wildlife Conservation (Permit No WL/3/2/13/15), IDEA WILD organization, the staff of Horton Plains National Park, the research crew of Wildlife Circle, Department of Zoology University of Sri Jayewardenepura and the Department of English University of Sri Jayewardenepura.

\section{References}

Ashton, M.S., Gunatilleke, S., De Zoysa, N., Dassanayake, M.D., Gunatilleke, N. and Wijesundera, S., 1997. A field guide to the common trees and shrubs of Sri Lanka. Colombo, Sri Lanka: WHT Publications.430pp.

DWC, 2005. Horton Plains National Park. Management Plan. Final Draft. Protected Areas Management and Wildlife Conservation Project, Department of Wildlife Conservation, Colombo. 91 pp.

DWC, 2007.Biodiversity Baseline Survey: Horton Plains National Park. Department of Wildlife Conservation, Ministry of Environment and Natural Resources, Colombo.40 pp. 
Green, M.J.B. and Gunawardena, E.R.N., 1997. Designing an optimum protected areas system for Sri Lanka's natural forests. 2 volumes. Environmental Management in Forestry Developments Project, Forest Department, Government of Sri Lanka, Colombo.399 pp.

Gunawardena,K. and Weerakoon, D.K. 2012. The Taxonomy and Conservation Status of Birds in Sri Lanka. Ministry of Environment, Colombo, Sri Lanka.114- 117.

Harrison, J. and Worfolk, T., 1999. A Field Guide to the Birds of Sri Lanka. Oxford University Press, Oxford. 219 pp.

Kaluthota, C. D. and Kotagama, S.W., 2009. Revised Avifaunal List of Sri Lanka. Occasional Paper No.02.Field Ornithology Group of Sri Lanka 32pp.

Kotagama, S. W., 1993. Wildlife conservation and development of the south east dry zone. In The Southeast dry Zone of Sri Lanka. Colombo: Agrarian Research and Training Institute.

Magurran, A. E., 1988. Ecological Diversity and its Measurement. Croom Helm Limited, London. 179 pp.

MfC, 1994. Research in Horton Plains National Park, Sri Lanka. Final Report sumitted to Biodiversity Support Programme. March for Conservation, Colombo. 68 pp.

MOE, 2012. The National Red List 2012 of Sri Lanka; Conservation Status of the Fauna and Flora. Ministry of Environment, Colombo, Sri Lanka. 476pp.

Myers, N., Mittermeier, R.A., Mittermeier, C.G., Da Fonseca, G.A. and Kent, J., 2000. Biodiversity hotspots for conservation priorities. Nature, 403. 853-858.

Pethiyagoda, R., 2012. Horton Plains Sri Lanka's Cloud-forest National Park, Wildlife Heritage Trust, Lake Crescent, Colombo 2, Sri Lanka. 320pp.

Sutherland, W.J., Newton, I. and Green, R., 2004. Bird ecology and conservation: a handbook of techniques (No. 1). Oxford University Press. 408pp.

WCMC, 1997. A Global Directory of Tropical Montane Cloud Forests .Aldrich, M., Billington, C, Edwards, M. and Laidlaw, R (Eds).World Conservation Monitoring Centre, Cambridge, UK. 268pp.

Williams, R.A., 2015. Mitigating biodiversity concerns in Eucalyptus plantations located in South China. Journal of Biosciences and Medicines, 3(06). 1-8.

World Bank, 2016. Forest area (\% of land area). Retrieved on (2016, March, 29) from http://data.worldbank.org/indicator/AG.LND.FRST.ZS 